\title{
Preoperative Differentiation Between Malignant and Benign Ovarian Masses in Patients with Normal CA-125 Levels
}

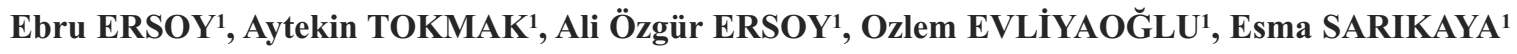 \\ Ankara, Turkey
}

\begin{abstract}
OBJECTIVE: We aimed to determine the diagnostic values of some clinical and ultrasonographic features for predicting ovarian malignancies in patients with normal serum CA-125 levels.

STUDY DESIGN: All of the 84 patients who were diagnosed with ovarian malignancy and 168 patients with benign adnexal mass had normal CA-125 levels, and recruited as study and control groups, respectively. Individual characteristics, ultrasonographic features, and risk of malignancy index (RMI) values of the groups were compared retrospectively.
\end{abstract}

RESULTS: The ages, mean postmenopausal period of the postmenopausal women in the malignant group were significantly higher than the benign group. Nulliparity rates were similar between the two groups. The number of postmenopausal patients was significantly higher in the malignant group. Median cyst diameter and RMI values were significantly higher, and bilaterality and presence of solid area were more frequent in this group. Logistic regression model showed that ovarian cysts larger than $8 \mathrm{~cm}$ [Odds ratio (OR): $7.012 ; p<0.001 ; 95 \%$ Confidence interval $(\mathrm{Cl}): 3.463-14.198]$, presence of solid area within the cyst [OR: $7.43 ; p<0.001 ; 95 \% \mathrm{Cl}: 3.799-14.532]$, and postmenopausal state [OR: $2.893 ; p=0.027$; $95 \% \mathrm{Cl}: 1.129-7.412]$ were found to be significant factors to predict malignancy.

CONCLUSIONS: Ovarian cysts larger than $8 \mathrm{~cm}$, presence of solid area within the cyst, and postmenopausal status are the most important risk factors for ovarian malignancies in these patients with normal CA-125 levels.

Keywords: CA-125, Histological type, Ovarian cancer, Risk of malignancy index, Tumor marker

Gynecol Obstet Reprod Med 2016;22(2):94-98 DOI: 10.21613/GORM.2016.80

\section{Introduction}

Gynecologic malignities still represent a major burden of death risk, despite novel diagnosis and treatment modalities. Ovarian malignancy is the most mortal type of gynecologic malignities (1). Many women with ovarian cancer are diagnosed in late stages of the disease. Unfortunately, the lack of effective screening methods for early diagnosis leads to this condition (2). In preoperative assessment, prediction of the benign or malignant character of an ovarian cyst is crucial and an accurate diagnosis is required for choosing an appropriate treatment method.

The most frequently used and effective diagnostic screening methods are serum CA-125 levels and ultrasonographic

${ }^{1}$ Department of Obstetrics and Gynecology Zekai Tahir Burak Women's Health Care Training and Research Hospital, Ankara

Address of Correspondence: Ebru Ersoy

Zekai Tahir Burak Women's Health Care Training and Research Hospital

Talatpasa Bulv. Hamamonu, Altındă̆

Ankara, Turkey

eebruersoy09@gmail.com

Submitted for Publication:

19. 04.2016

Accepted for Publication: features of the cyst. CA-125 is a glycoprotein which takes its source from embryonic coelomic epithelium. Its predictability about malignancy takes its place in especially epithelial ovarian tumors. On the other hand, it can be also found increased in circumstances of some benign conditions such as endometriosis, pregnancy, cirrhosis (3).

In the past, postmenopausal ovarian mass and palpable ovaries were the major indication for the surgery in suspicion of ovarian cancer. The increasingly widespread use of ultrasound has increased the number of patients diagnosed with ovarian cyst. However, the extent of unnecessary surgery for ovarian cysts has been also avoided by identification of some specific ultrasonographic features. Recently, different laboratory markers, imagination methods and clinical parameters were presented for identifying malignancy potential of an ovarian mass. Risk of malignancy index (RMI), which is one of them, is commonly utilized to discriminate the at-risk group. It is based on ultrasonographic signs, serum CA-125 level, and menopausal status (4).

Abovementioned screening modalities are readily available in most gynecologic clinics, if we consider them instead of other sophisticated laboratorial and molecular analyses. We aimed to investigate which patients are at-risk group even if the RMI and CA-125 levels were all in normal ranges. 


\section{Material and Method}

This retrospective study was conducted at the Department of Gynecology of our institution in Ankara, Turkey. The study institution is a tertiary referral center for gynecology. The study had been approved by the institutional review board, before we accessed to medical records (approval date/number: 27.02.2015/25). Owing to the retrospective study design, informed consent was not needed.

The study was designed by using a 1:2 ratio for cases:controls. A total of 84 consecutive patients who were diagnosed with gynecological malignancy finally, and 168 patients as a control group who were diagnosed with benign ovarian masses according to final pathologic reports were included. Borderline types were merged into malignant group. All included participants who attended to the institution for the surveillance of an ovarian mass from January 2011 to December 2013, had normal RMI (<200) (5), and serum CA-125 levels $(<35 \mathrm{IU} / \mathrm{mL})(6)$, and both groups were matched for CA-125 levels. Exact diagnoses and laboratory results were obtained and confirmed from medical records. The inclusion criteria were as follows: non-pregnant woman, undergone oophorectomy or mass excision due to the persistent symptoms of pain localized to lower abdominal quadrants and/or malignancy suspicion. Exclusion criteria were patients had missing medical records, extraovarian malignancy, and ovarian metastases from other gynecologic or non-gynecologic organs. Patients were also excluded if any of the following disorders that would affect serum CA-125 levels (even within normal ranges) were present: a history of or current pelvic inflammatory disease, previous pelvic surgery, endometriosis, myoma uteri, adenomyosis, endometrial pathology, acute infection or history of chronic inflammatory disease, metabolic and autoimmune disorders.

The ages, previous reproductive history, serum CA-125 levels and ultrasonographic findings of the ovarian masses were obtained from medical records. RMI was calculated for each patient using the following formula: Ultrasound score $\mathrm{x}$ Menopause score x CA-125 level (5). Ultrasound score was calculated as follows: each one of present bilaterality, solid areas, multilocularity, ascites, intraabdominal metastases criteria was evaluated as one point, the ultrasound score was defined as the summation of these. Menopause score was calculated as follows: amenorrhea lasting for longer than one year, an age of 50 years or older, a postmenopausal status was accepted as 3 points of menopause score. A premenopausal status was accepted as one point of menopause score (5).

For all patients, ultrasonographic examinations were performed by expert radiologists of our institution with the use of Aloka Prosound alpha 7 (Hitachi Aloka Medical, Tokyo, Japan), Aloka Prosound SSD 5500 (Aloka Inc, Tokyo, Japan), Toshiba Aplio 500 (Toshiba Medical Systems Corporation, Tokyo, Japan), and Hitachi Avius (Hitachi Medical, Tokyo,
Japan) ultrasonographic systems equipped with abdominal probe $(3.5-5 \mathrm{MHz})$ or transvaginal probe $(5-9 \mathrm{MHz})$. Tumoral size was determined using mean diameter of the mass. Staging of ovarian malignity was performed with the use of FIGO Ovarian Cancer Staging system (7). Histopathological typing of each patient's specimen was performed by using the World Health Organization's classification of tumors (8). Serum CA125 levels were measured using an electrochemiluminescence immunoassay method (Roche Elecsys kits; Roche Diagnostics, Mannheim, Germany), and concentrations were expressed as $\mathrm{IU} / \mathrm{mL}$. It was noted that all blood analyses were done during the early follicular phase in women still menstruating.

For records and statistical analyses, Statistical Package for Social Sciences (SPSS, Chicago, IL, USA) version 15.0 for Windows was used. Distribution of the data was analyzed with Kolmogorov-Smirnov and Shapiro-Wilk test. The data were presented as mean with standard deviation or median with interquartile ranges for continuous variables, and as number with percentage for categorical variables. In comparisons between groups, Independent samples $\mathrm{T}$ test was used for parametric variables and Mann-Whitney $U$ test was used for nonparametric variables. Proportions were compared with the Chi-square test or Fisher's exact test. ROC analysis was performed to determine the optimal cut off values. Binomial logistic regression analysis was used to identify independent risk factors for ovarian malignities. In all analyses, two-tailed p-values of $<0.05$ were considered statistically significant.

\section{Results}

Ages of the patients ranged between 19-68 years, and 1982 years; CA125 levels 2.18-34.6 IU/mL, and 4.4-35 IU/mL; RMI values 2.6-195.6, and 4.4-198.5 in benign and malignant groups, respectively. These variables in the malignant group were significantly higher than in the benign group. Mean postmenopausal period of the 35 postmenopausal patients in the malignant group (11.8 \pm 8.8 years) was significantly higher than of the 29 postmenopausal patients in the benign group $(6.3 \pm 4.4$ years $)(\mathrm{p}=0.011)$. Comparison of the individual characteristics of the groups was shown in table 1.

The two most frequent stages were 1a (49 patients, $58.3 \%$ ), and 1c (19 patients, 22.6\%). The followings were stages of $2 \mathrm{a}$ ( 5 patients, $6.9 \%$ ), $1 \mathrm{~b}$ ( 4 patients, $4.8 \%$ ), 3c (4 patients, $4.8 \%$ ), 4 (2 patients, $2.4 \%$ ), and $3 \mathrm{a}$ (one patient, $1.2 \%$ ), respectively. Serous cystadenoma was the most frequent type in the benign group. Granulosa cell type was the most frequent histological type in the malignant group. The histological types of the two groups were shown in table 2 .

The cutoff values for age and mass diameter to differentiate between malignant and benign cysts were found to be 45 years and $8 \mathrm{~cm}$, respectively. In multivariate binomial logistic regression analysis; ovarian cysts larger than $8 \mathrm{~cm}$, presence of solid area within the cyst, and menopausal status are the in- 
dependent risk factors for ovarian malignancies in patients with normal serum CA 125 levels (Table 3).

\section{Discussion}

Ovarian cancer is known to be a sneaky cancer because the symptoms are often vague, and can be easily confused with gastrointestinal disorders such as gastritis, bowel gas, and irritable bowel syndrome. The mortality rate of ovarian cancer is the highest among all gynecological cancers due to delayed diagnosis. Therefore, early diagnosis of ovarian cancer saves lives. Although available data suggests that high serum CA125 levels are detected in the majority of ovarian cancers prior to clinical presentation, as a screening method for ovarian cancer, CA-125 has a high sensitivity and low specificity for detecting early disease (9). The preoperative diagnosis of ovarian cancer sometimes remains challenging, particularly in those with normal CA-125 values.

Table 1: Comparison of the individual characteristics of the groups

\begin{tabular}{lllr}
\hline Age (years) & $47.6 \pm 15.3$ & $40.1 \pm 11.0$ & $<\mathbf{0 0 1}$ \\
Nulliparity $\mathrm{n}(\%)$ & $16(19)$ & $38(22.6)$ & 0.515 \\
Menopause score>1 $\mathrm{n}(\%)$ & $36(41.7)$ & $29(17.3)$ & $<\mathbf{0 0 1}$ \\
USG score>1 $\mathrm{n}(\%)$ & $21(25)$ & $7(4.2)$ & $<\mathbf{0 0 1}$ \\
$\quad$ Bilaterality & $10(11.9)$ & $8(4.8)$ & $\mathbf{0 . 0 3 8}$ \\
$\quad$ Ascites & $2(2.4)$ & $1(0.6)$ & 0.255 \\
$\quad$ Solid area & $59(70.2)$ & $42(25)$ & $<0.001$ \\
$\quad$ Multilocularity & $18(16.1)$ & $27(21.4)$ & 0.295 \\
CA125 median \pm IQR (IU/mL) & $17.5 \pm 13.9$ & $15.3 \pm 12.3$ & 0.189 \\
MD median \pm IQR (mm) & $90.5 \pm 64.5$ & $62 \pm 29.7$ & $<0.001$ \\
RMI median \pm IQR & $28.3 \pm 32.8$ & $18.4 \pm 15.5$ & $<0.001$ \\
\hline
\end{tabular}

IQR: Interquartile range, USG: Ultrasound, MD: Mass diameter, RMI: Risk of malignancy index. $p<0.05$ is statistically significant

Table 2: The histological types of the two groups

\begin{tabular}{l|c|c|c}
\hline \multicolumn{2}{|c|}{ Malignant group } & & \multicolumn{2}{c}{ Benign group } \\
\hline Histological type & $\mathbf{n ~ ( \% )}$ & Histological type & $\mathbf{n}(\%)$ \\
\hline Borderline serous & $16(19.0)$ & Serous cystadenoma & Mucinous cystadenoma \\
Borderline mucinous & $6(7.1)$ & Dermoid cyst & $24(14.3)$ \\
Serous carcinoma & $6(7.1)$ & Haemorrhagic cyst & $40(23.8)$ \\
Mucinous carcinoma & $8(9.5)$ & Follicle cyst & $18(10.7)$ \\
Endometrioid & $10(11.9)$ & Fibroma & Total \\
Clear cell carcinoma & $6(7.1)$ & & $7(4.2)$ \\
Carcinosarcoma & $2(2.4)$ & & $168(100)$ \\
Dysgerminoma & $1(1.2)$ & & \\
Lymphoma & $1(1.2)$ & & \\
Granulosa cell & $20(23.8)$ & & \\
Fibrosarcoma & $1(1.2)$ & & \\
Sex cord-stromal & $2(2.4)$ & & \\
Mixed tumor & $2(2.4)$ & & \\
Schwannoma & $1(1.2)$ & & \\
Sertoli - Leydig & $2(2.4)$ & & \\
Total & $84(100.0)$ & & \\
\hline
\end{tabular}

Table 3: Multivariate binomial logistic regression analysis showing the predictive values of individual risk factors for malignancy

\begin{tabular}{lllll}
\hline Outcome: Malignancy & Wald & $\mathrm{p}$ & $\mathrm{OR}$ & $95 \% \mathrm{Cl}$ \\
\hline Solid area & 34.339 & $<0.001$ & 7.430 & $3.799-14.532$ \\
Postmenopausal state & 4.894 & $\mathbf{0 . 0 2 7}$ & 2.893 & $1.129-7.412$ \\
Bilaterality & 1.047 & 0.306 & 1.881 & $0.561-6.309$ \\
Age $>$ 45 years & 1.348 & 0.246 & 1.677 & $0.701-4.012$ \\
MMD $>8 \mathrm{~cm}$ & 29.280 & $<0.001$ & 7.012 & $3.463-14.198$ \\
\hline
\end{tabular}

Cl: Confidence interval, MMD: Median mass diameter 
In this study, we aimed to determine the diagnostic values of some clinical and ultrasonographic features for predicting ovarian malignancies in patients with normal serum CA-125 levels. According to the study, ovarian cysts larger than $8 \mathrm{~cm}$, presence of solid area within the cyst, and menopausal status are the most important risk factors for ovarian malignancies independently of CA-125 and RMI values.

Ultrasonography is a simply applicable, safe and inexpensive diagnostic method used in gynecology practice. CA-125 and ultrasonographic criteria were found useful to predict ovarian malignancies $(10,11)$. However, a normal CA-125 level does not rule out ovarian cancer, up to $50 \%$ of early stage ovarian malignities and $20 \%$ to $25 \%$ of advanced cancers have been reported to have normal levels of CA-125 (12). For this reason, Jacobs et al. originally developed a RMI for predicting whether there is a malignancy in women presenting with an ovarian or pelvic mass. They produced a formula consisting of serum CA-125 level, menopause status, and ultrasound scan result. In several subsequent studies, RMI was confirmed to be highly significant marker for predicting ovarian malignities. A study from Brazil compared 4 different RMI models, and found them to have similar efficacy and success rates. The positive likelihood ratios of the 4 RMI variants were reported to range from 3.52 to 4.41 (13).

Although many modified methods and new scoring systems have been defined in the preoperative assessment of adnexal mass, RMI is still recommended in both the UK and USA (14). Similarly, we showed that advanced age, menopausal status, having a mass with complex ultrasonographic features such as solid area, bilaterality, and elevated mass diameter were associated with malignancy as well as RMI; despite these patients have normal CA-125 levels. Moreover, advanced postmenopausal period was found to be related with malignancy.

Ovarian malignities may occur at all ages with the variation in histological subtype by age. Germ cell tumors are commonly seen in younger women, while borderline tumors generally occur in 30-40 years of age, and epithelial ovarian cancer is seen after the 50 years (15). It was suggested that ovarian masses in postmenopausal women should be evaluated carefully, because ovarian tumors in postmenopausal women were mostly neoplastic (16). In our study epithelial and borderline tumors were more common than the other histological types. The number of postmenopausal patients was also significantly higher in the malignant group. Therefore, we found higher rates of malignancy in women older than 45 years.

In reproductive age women, ovarian masses greater than 8 to $10 \mathrm{~cm}$ are commonly explored. Smaller simple cysts (5 to $8 \mathrm{~cm}$ ) should be managed conservatively with a repeat examination in 2 months, because most of these cysts are functional and are resolved spontaneously in 4 to 8 weeks. Okugawa et al. showed that the mean maximum size was 7.1 $\mathrm{cm}$ in tumor like lesions (such as endometriosis), $10.9 \mathrm{~cm}$ in benign tumors, $13.6 \mathrm{~cm}$ in malignant tumors, and $18.5 \mathrm{~cm}$ in low malignant potential (LMP) tumors in their retrospective review of 1648 cases (17). Koonings et al. found the mean size of benign ovarian tumors to be $(8-10 \mathrm{~cm})$, slightly smaller than that of malignant tumors $(11-14 \mathrm{~cm})$, and tumors of LMP (10-12 cm) (16). Rulin et al. reported that $64 \%$ of adnexal masses greater than $10 \mathrm{~cm}$ in diameter were malignant, while only 1 out of 32 cases was malignant in the less than $5 \mathrm{~cm}$ group among postmenopausal women (18). In our study, women with malignant mass had larger mass diameter than those with benign mass. The median mass diameter was about $9 \mathrm{~cm}$ in malignant cases, and we found that an ovarian mass larger than $8 \mathrm{~cm}$ was a significant risk factor for ovarian cancer.

Another study of Koonings et al. showed that women with bilateral ovarian neoplasms had a two-fold increased risk of malignancy when compared with women who had unilateral neoplasms (19). In our study, bilaterality was significantly more common in the malignant group. This finding is in accordance with the literature.

High prevalence of the patients who were diagnosed with stage 1a and 1c ovarian malignancy can be attributable to higher ratios of early diagnosis. Owing to being a tertiary care center, we thought that ovarian masses can be diagnosed with great accuracy in our hospital. Consistent with the literature, serous cystadenoma was the most frequent neoplasia among the benign final pathologies (20). The low prevalence of the benign functional pathologies (follicle cyst, haemorrhagic cyst) can be attributable to lower incidences of surgical therapy in the management of these pathologies.

The main limitation of this study is to be designed as a retrospective study. Ultrasonographic evaluations were made by different radiologists using different ultrasonographic systems. The study covered a very wide age range of patients. Therefore final histopathologic types were different among the patients. However, as far as we know, this study is the first comparing clinical and ultrasonographic features of patients with normal CA-125 levels according to their malignancy status.

In conclusion, ovarian malignancies in patients with normal CA 125 values are frequently diagnosed in early stages of the disease. Ovarian cysts larger than $8 \mathrm{~cm}$, presence of solid are within the cyst, and menopausal status are the most important risk factors for ovarian malignancies in these patients. A careful ultrasonographic evaluation in postmenopausal women with large ovarian mass may contribute to the early detection of ovarian cancer, despite having a normal CA125 level.

\section{References}

1. Walker JL, Powell CB, Chen LM, et al. Society of Gynecologic Oncology recommendations for the preven- 
tion of ovarian cancer. Cancer 2015;121:2108-120.

2. Simsek HS, Tokmak A, Ozgu E, et al. Role of a risk of malignancy index in clinical approaches to adnexal masses. Asian Pac J Cancer Prev 2014;15:7793-7.

3. Oei AL, Massuger LF, Bulten J, Ligtenberg MJ, Hoogerbrugge N, de Hullu JA. Surveillance of women at high risk for hereditary ovarian cancer is inefficient. $\mathrm{Br} \mathrm{J}$ Cancer 2006;94:814-9.

4. Terzic M, Dotlic J, Likic I, et al. Risk of malignancy index validity assessment in premenopausal and postmenopausal women with adnexal tumors. Taiwan J Obstet Gynecol 2013;52:253-7.

5. Jacobs I, Oram D, Fairbanks J, Turner J, Frost C, Grudzinskas JG. A risk of malignancy index incorporating CA 125, ultrasound and menopausal status for the accurate preoperative diagnosis of ovarian cancer. Br J Obstet Gynaecol 1990;97:922-9.

6. Bast RC Jr, Klug TL, St John E, et al. A radioimmunoassay using a monoclonal antibody to monitor the course of epithelial ovarian cancer. N Engl J Med 1983;309:883-7.

7. Berek JS, Crum C, Friedlander M. Cancer of the ovary, fallopian tube, and peritoneum. Int J Gynaecol Obstet 2012;119:S118-29.

8. World Health Organization classification of tumours. Pathology and genetics of the breast and female genital organs. Lyon, IARC Press 2003

9. Rosenthal AN, Jacobs IJ. The role of CA 125 in screening for ovarian cancer. Int J Biol Markers 1998;13:216-20.

10. Kaymak O, Ustunyurt E, Okyay RE, Kalyoncu S, Mollamahmutoglu L. Role of Transvaginal Sonography and Serum CA-125 Values in Determining the Nature of the Postmenopausal Ovarian Cysts. J Turk Ger Gynecol Assoc 2006;7:24-28.

11. Basogul N, Sanci M, Tinar S, Basogul O, Inal MM. The Importance of Doppler Ultrasonography, Ultrasonograp- hic Morphologic Scores and Ca-125 in Differential Diagnosis of Pelvic Masses. Gynecol Obstet Reprod Med 2000;6(3):201-204.

12. American College of Obstetricians anf Gynecologists. ACOG Committee Opinion: number 280, December 2002. The role of the generalist obstetrician-gynecologist in the early detection of ovarian cancer. Obstet Gynecol 2002;100:1413-6.

13. Campos C, Sarian LO, Jales RM, et al. Performance of the Risk of Malignancy Index for Discriminating Malignant Tumors in Women With Adnexal Masses. J Ultrasound Med 2016;35:143-52.

14. Geomini P, Kruitwagen R, Bremer GL, Cnossen J, Mol $\mathrm{BW}$. The accuracy of risk scores in predicting ovarian malignancy: a systematic review. Obstet Gynecol 2009; 113:384-94.

15. Berek JS, Crum C, Friedlander M. Cancer of the ovary, fallopian tube, and peritoneum. Int $\mathrm{J}$ Gynaecol Obstet 2015;131 Suppl 2:S111-22.

16. Koonings PP, Campbell K, Mishell DR Jr, Grimes DA. Relative frequency of primary ovarian neoplasms: a 10year review. Obstet Gynecol 1989;74:921-6.

17. Okugawa K, Hirakawa T, Fukushima K, Kamura T, Amada S, Nakano H. Relationship between age, histological type, and size of ovarian tumors. Int J Gynaecol Obstet 2001;74:45-50.

18. Rulin MC, Preston AL. Adnexal masses in postmenopausal women. Obstet Gynecol 1987;70:578-81.

19. Koonings PP, Grimes DA, Campbell K, Sommerville M. Bilateral ovarian neoplasms and the risk of malignancy. Am J Obstet Gynecol 1990;162:167-9.

20. Kanthikar SN, Dravid NV, Deore PN, Nikumbh DB, Suryawanshi KH. Clinico-Histopathological Analysis of Neoplastic and Non-Neoplastic Lesions of the Ovary: A 3Year Prospective Study in Dhule, North Maharashtra, India. J Clin Diagn Res 2014;8:FC04-7. 